\title{
The efficacy of glycemic control with continuous glucose monitoring on atheroma progression: rationale and design of the Observation of Coronary Atheroma Progression under Continuous Glucose Monitoring Guidance in Patients with Type 2 Diabetes Mellitus (OPTIMAL)
}

\author{
Yu Kataoka ${ }^{1}$, Kiminori Hosoda ${ }^{2}$, Hisashi Makino², Masaki Matsubara ${ }^{2}$, Miki Matsuo ${ }^{2}$, Yoko Ohata ${ }^{2}$, \\ Ryo Koezuka ${ }^{2}$, Tamiko Tamanaha ${ }^{2}$, Tsutomu Tomita ${ }^{2}$, Kyoko Honda-Kohmo ${ }^{2}$, Michio Noguchi ${ }^{2}$, \\ Cheol Son ${ }^{2}$, Kunihiro Nishimura ${ }^{3}$, Yasuhide Asaumi ${ }^{1}$, Yoshihiro Miyamoto ${ }^{4}$, Teruo Noguchi ${ }^{1}$, Satoshi Yasuda ${ }^{1}$ \\ ${ }^{1}$ Department of Cardiovascular Medicine, ${ }^{2}$ Division of Atherosclerosis and Diabetes, ${ }^{3}$ Department of Statistics and Data Analysis, ${ }^{4}$ Division of \\ Preventive Cardiology, National Cerebral \& Cardiovascular Center, Osaka, Japan \\ Contributions: (I) Conception and design: Y Kataoka, H Makino, K Hosoda; (II) Administrative support: K Hosoda; (III) Provision of study materials \\ or patients: Y Kataoka, H Makino, K Hosoda; (IV) Collection and assembly of data: Y Kataoka, H Makino; (V) Data analysis and interpretation: K \\ Nishimura; (VI) Manuscript writing: All authors; (VII) Final approval of manuscript: All authors. \\ Correspondence to: Kiminori Hosoda, MD, PhD. Division of Atherosclerosis and Diabetes, National Cerebral \& Cardiovascular Center, Fujishirodai \\ 5-7-1, Suita, Osaka, Japan. Email: kiminorihosoda@ncvc.go.jp.
}

Background: Patients with type 2 diabetes mellitus (T2DM) are high-risk subjects who more frequently have micro- and macrovascular diseases including coronary artery disease (CAD). Since impaired glycemic homeostasis directly influences the formation and propagation of atherosclerotic plaques, optimal management of glycemic status is required for the prevention of diabetic atherosclerosis. Continuous glucose monitoring (CGM) provides not only average glucose level but also the degree of glucose fluctuation and hypoglycemia. Given the association of glycemic variability with diabetic macrovascular diseases, CGMbased glycemic management could favorably modulate glycemic fluctuation, thereby potentially modifying atheroma burden in T2DM subjects. To test this hypothesis, the Observation of Coronary Atheroma Progression under Continuous Glucose Monitoring Guidance in Patients with Type 2 Diabetes Mellitus (OPTIMAL) study has been designed (Japan Registry of Clinical Trials: jRCT1052180152, University Hospital Medical Information Network Clinical Trial Registry UMIN000036721).

Methods: The OPTIMAL is a single-center, randomized trial to evaluate the efficacy of CGM-based glycemic control on atheroma progression in T2DM patients with CAD by using serial intravascular ultrasound (IVUS) and near-infrared spectroscopy (NIRS) imaging. A total of 90 eligible subjects will be randomized 1:1 into two groups to receive either CGM-based glycemic control or HbA1c-baded glycemic management. Coronary angiography and NIRS/IVUS imaging is repeated at the end of the assigned treatment period.

Results: The primary endpoint is the normalized absolute change in total atheroma volume (TAV) from baseline to 12 months. The secondary endpoints include (I) the absolute change in percent atheroma volume, (II) the percent change in lipid core burden index, (III) the change in coefficient variance measured by CGM, (IV) the change in atherogenic markers (high-density lipoprotein functionality, proprotein convertase subxilisin/kexin type 9 and fatty-acid binding proteins), and (V) the frequency of hypoglycemia. Safety will also be evaluated.

Conclusions: The collaboration of CGM use with serial NIRS/IVUS imaging will enable to compare atheroma progression rate under CGM-based glycemic management and HbA1c-based approach. 


\begin{abstract}
Keywords: Type 2 diabetes mellitus (T2DM); coronary atherosclerosis; glucose; continuous glucose monitoring
\end{abstract} (CGM); glycated hemoglobin; intravascular ultrasound (IVUS); near-infrared spectroscopy (NIRS)

Submitted Jun 19, 2019. Accepted for publication Aug 13, 2019.

doi: $10.21037 / \mathrm{cdt} .2019 .09 .02$

View this article at: http://dx.doi.org/10.21037/cdt.2019.09.02

\section{Introduction}

Current therapeutic guidelines recommend achieving glycated hemoglobin $\mathrm{HbA} 1 \mathrm{c}<7.0 \%$ to prevent macrovascular diseases in type 2 diabetes mellitus (T2DM) patients $(1,2)$. However, several randomized controlled trials demonstrated that stricter control of HbA1c did not necessarily improve their cardiovascular outcomes (3-5). In particular, intensive lowering HbA1c elevates a risk of hypoglycemia which has been shown to associate with higher mortality (5). These observations suggest the limitation of HbAlc-based glycemic control for the prevention of diabetic macrovascular diseases. Given that $\mathrm{HbA1c}$ reflects average blood glucose in the past 1-2 months but not serial changes in glucose level including hypoglycemia, the another approach enabling real-time accurate monitoring of glucose level is required.

Continuous glucose monitoring (CGM) continuously evaluates glucose levels in the interstitial fluid, which provides not only average glucose level but also the degree of glucose fluctuation and hypoglycemia (6). Since glucose fluctuation and hypoglycemia have been shown to induce pro-atherogenic responses (7), we hypothesize that CGMbased glycemic management could favorably modulate glycemic fluctuation, which slows progression of coronary atherosclerosis, an important substrate for macrovascular disease in T2DM subjects. Therefore, the current study is designed to evaluate the effect of glucose control with CGM guidance on coronary atherosclerosis in T2DM patients with $\mathrm{CAD}$ and compare with HbA1c-based glycemic control by using serial IVUS/NIRS imaging, which quantitatively evaluate coronary atherosclerosis in vivo.

\section{Methods}

\section{Study design}

OPTIMAL is an investigator-initiated, prospective, randomized, parallel-group, single center trial (jRCT1052180152, UMIN000036721) (Table S1). This study evaluates the efficacy of glucose lowering under CGM guidance on progression of coronary atherosclerosis in T2DM patients with coronary artery disease (CAD) who will require percutaneous coronary intervention (PCI). Enrollment of 90 patients is planned at National Cerebral \& Cardiovascular Center in Japan.

T2DM patients hospitalized for stable CAD requiring PCI are potentially eligible. Screening is performed prior to the scheduled PCI. In patients who fulfill all eligibility criteria, written informed consent will be obtained by primary or co-investigators. Study participants are randomly assigned to either CGM-based glucose management or HbA1c-based glucose management. Randomization is stratified according to age, gender, high-intensity statin use, HbA1c and LDL-C levels by co-investigator (Nishimura $\mathrm{K})$. Randomization will be stratified by age, gender, HbA1c, LDL-C and high-intensity statin use. Clinical visits are scheduled at 1, 3, 6, 9 and 12 months after the completion of PCI. In the CGM-based glucose management group, CGM and HbA1c measurement will be conducted at baseline and 3, 6, 9 and 12 months following PCI. In the HbA1c-based glucose management group, CGM will be done at baseline and 12 months in a similar fashion. The study schedule is summarized by Table 1 .

\section{Inclusion and exclusion criteria}

Inclusion criteria are summarized in Table 2. Briefly, eligible subjects should have CAD requiring elective PCI. Mild stenosis needs to exist within non-culprit major coronary artery. Since the current study enroll subjects who need glucose lowering therapy to achieve HbA1c $<7.0 \%$, HbA1c at screening should be between $7.0 \%$ and $10.0 \%$. In T2DM subjects who already receive sulphonylurea, nateglinide and/or insulin at screening, baseline HbA1c should be below $10.0 \%$ and the lowest cut-off is not set. Due to an elevated risk of hypoglycemia under these anti-diabetic agents, subjects with HbA1c < $7.0 \%$ may have hypoglycemic events which require adequate glycemic control.

The main exclusion criteria are listed in Table 3. Nonculprit vessel with its severe tortuousty and/or calcification 
Table 1 The schedule of enrolment, interventions, and assessments

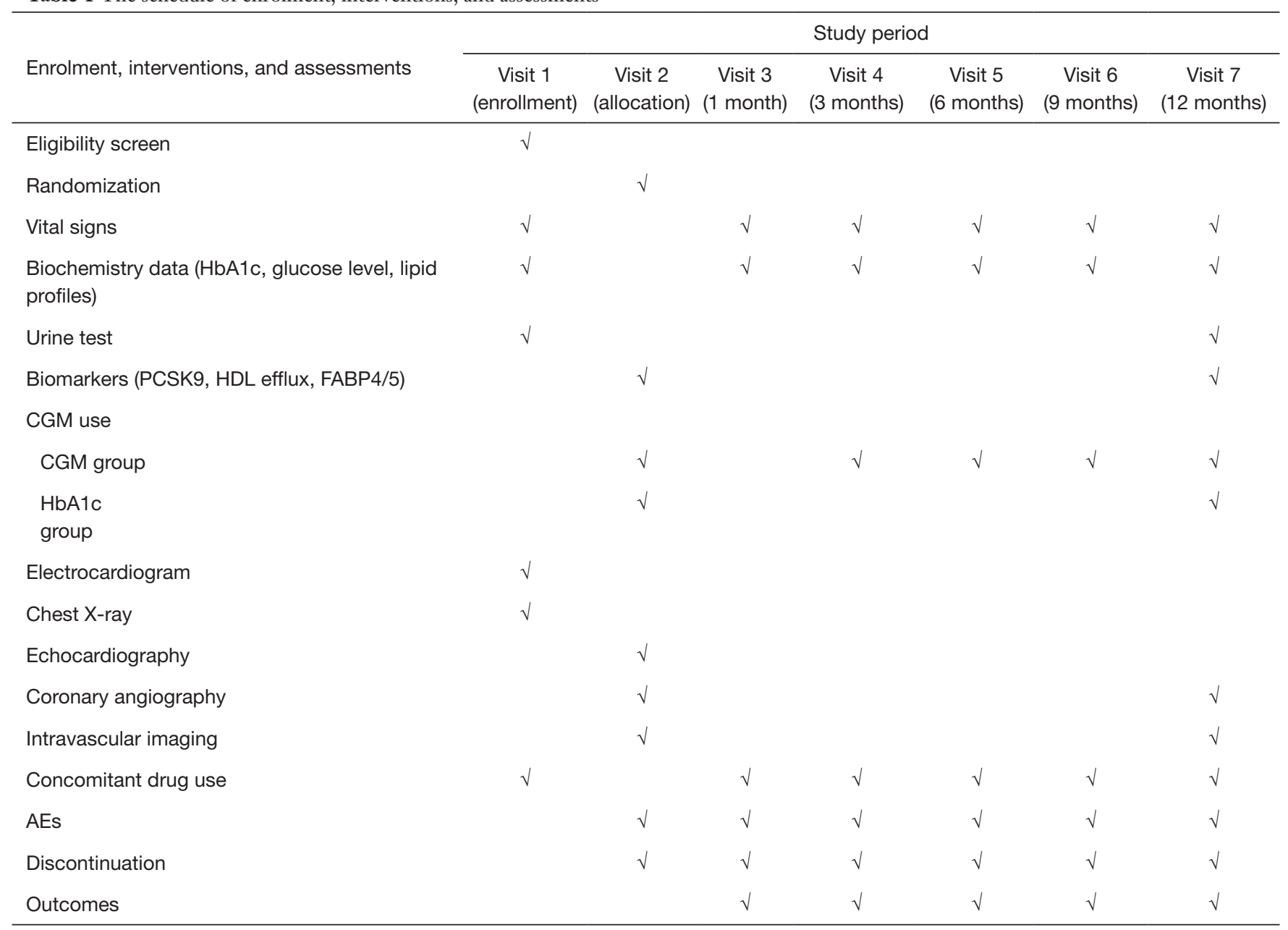

CGM, continuous glucose monitoring; AEs, adverse effects.

Table 2 Inclusion criteria

Male of female between 20 and 80 years of age

Type 2 diabetic patients with coronary artery disease who require $\mathrm{PCl}$

The presence of mild stenosis in the non-target vessel (\% diameter stenosis between 10-50\%)

$7.0 \% \leq \mathrm{HbA} 1 \mathrm{c} \leq 10.0 \%$

$\mathrm{HbA} 1 \mathrm{c} \leq 10.0 \%$ in subjects who receive insulin, sulfonylurea or nateglinide

Ability to understand the requirements of the study and to provide informed consent

$\mathrm{HbA} 1 \mathrm{c}$, glycated hemoglobin; $\mathrm{PCl}$, percutaneous coronary intervention.
Table 3 Exclusion criteria

Very tortuous coronary artery and/or severe calcification which is unsuitable for intravascular imaging

Subjects with severe renal dysfunction (estimated glomerular filtration rate $<40 \mathrm{~mL} / \mathrm{min} / 1.73 \mathrm{~m}^{2}$ )

The absence of any atherosclerotic lesions in the non-target vessel

Those who take PCSK9 inhibitor

Current enrolment in another investing device or drug study

Pregnancy

PCSK9, proprotein convertase subxilisin/kexin type 9. 
will be excluded due to the risk of NIRS/IVUS imaging. Subjects with baseline estimated glomerular filtration rate $<40 \mathrm{~mL} / \mathrm{min} / 1.73 \mathrm{~m}^{2}$, those without any atherosclerotic lesions in their non-culprit vessels and patients who are taking or are scheduled to receive proprotein convertase subxilisin/kexin type 9 will not be eligible.

\section{Treatments and key procedures}

After informed consent has been obtained, elective PCI will be conducted to treat culprit lesion after dual antiplatelet therapy consisting of aspirin and a $\mathrm{P} 2 \mathrm{Y} 12$ inhibitor is commenced at least one week prior to PCI. All procedural decisions including the selection of device and adjunctive pharmacotherapy will be made according to the discretion of the individual PCI operator. Following the completion of PCI, guidewire will be inserted into the non-culprit vessel. NIRS/IVUS imaging will be advanced into the distal end of the non-culprit vessel artery and then automatically pullbacked at $2 \mathrm{~mm} / \mathrm{sec}$. The non-culprit vessel should have mild stenosis with percent diameter stenosis between $10-50 \%$.

Dual antiplatelet therapy will be continued at maintenance doses (aspirin $\leq 100 \mathrm{mg} /$ day and clopidogrel $75 \mathrm{mg} /$ prasugrel $3.75 \mathrm{mg} /$ ticagrelor $90 \mathrm{mg}$ ) for a duration that is determined by treating physicians, typically lifelong for aspirin and 6-12 months for clopidogrel/prasugrel/ ticagrelor. Guideline-recommended medical therapy including beta-blockers and angiotensin converting enzyme inhibitors/angiotensin receptor blockers will be administered as appropriate. Adequate intensity of statin is used to achieve LDL-C below $70 \mathrm{mg} / \mathrm{dL}$ according to the Japan Atherosclerosis Society therapeutic guideline. Other lipid lowering therapies are allowed to use if LDL-C therapeutic goal is not achieved under statin therapy. PCSK9 inhibitor is prohibited in the current study.

As mentioned above, in the CGM-based glucose management group, CGM (FreeStyle Libre Pro ${ }^{\circledR}$, Abbott, Chicago, Illinoi, the United States) and HbA1c measurement will be undertaken at baseline and 3, 6, 9 and 12 months following PCI. In the HbAlc-based glucose management group, $\mathrm{HbA1c}$ will be measured at baseline and 3, 6, 9 and 12 months after PCI, and CGM will be used at baseline and 12 months in a similar fashion (Figure 1).

With regard to the use of anti-diabetic drugs, in the CGM-guided glycemic control group, endocrinologist will select glucose lowering drugs to fulfill the following CGMderived goals: (I) the frequency of hypoglycemia $=0 \%$,
(II) the coefficient of variation $<36 \%$ and (III) averaged glucose level between $70-180 \mathrm{mg} / \mathrm{dL}$ (6). If the frequency of hypoglycemia is over $10 \%$ and/or the averaged glucose level is more than $400 \mathrm{mg} / \mathrm{dL}$, patients will be asked to visit within 1 month after CGM measurement. In the HbA1cguided therapy group, the selection of glucose lowering agents will be made according to the discretion of each endocrinologist to achieve HbAlc $<7.0 \%$.

At 12 months following PCI, patients will be hospitalized to take follow-up coronary angiography and intravascular imaging study. NIRS/IVUS imaging in the non-culprit vessel will be conducted again in a similar fashion.

Biomarkers including proprotein convertase subxilisin/ kexin type 9 , fatty-acid binding protein 4 and 5 , and caspase- 3 and myeloperoxidase will be measured at baseline and 12 months.

\section{Acquisition and analysis of NIRS/IVUS imaging}

In all cases, operators will be encouraged to maximize the length of NIRS-IVUS pullback performed. The analyzed segment will be defined by the proximal and distal fiduciary sites. Technicians blinded to the treatment status of the patient and the timing of each individual pullback will perform the analysis. A matched arterial segment will be determined for measurement that contains the same proximal and distal side branch. Cross-sectional images, spaced $1 \mathrm{~mm}$ apart, will be selected for measurement. The leading edges of the lumen and the external elastic membrane (EEM) will be defined by manual planimetry. In each image, the plaque area will be calculated as the area between the two leading edges. The total atheroma volume (TAV) is calculated as the summation of plaque areas in each measured cross-sectional image within the segment, and subsequently normalized by the median number of images analyzed in the entire cohort to account for heterogeneity in segment length between subjects.

With regard to NIRS analysis, spectroscopic information obtained from raw spectra will be transformed into a probability of lipid core that will be mapped to a red-to-yellow color scale, with the low probability of lipid shown as red and the high probability of lipid shown as yellow. Analyses will be performed offline using the commercially available software (QIvus ${ }^{\circledR}$, Medis, Leiden, The Netherland). Yellow pixels within the analyzed segment will be divided by all viable pixels to generate the lipid-core burden index (LCBI). The maximal value of LCBI for the analyzed segment will be recorded and used for comparison. 


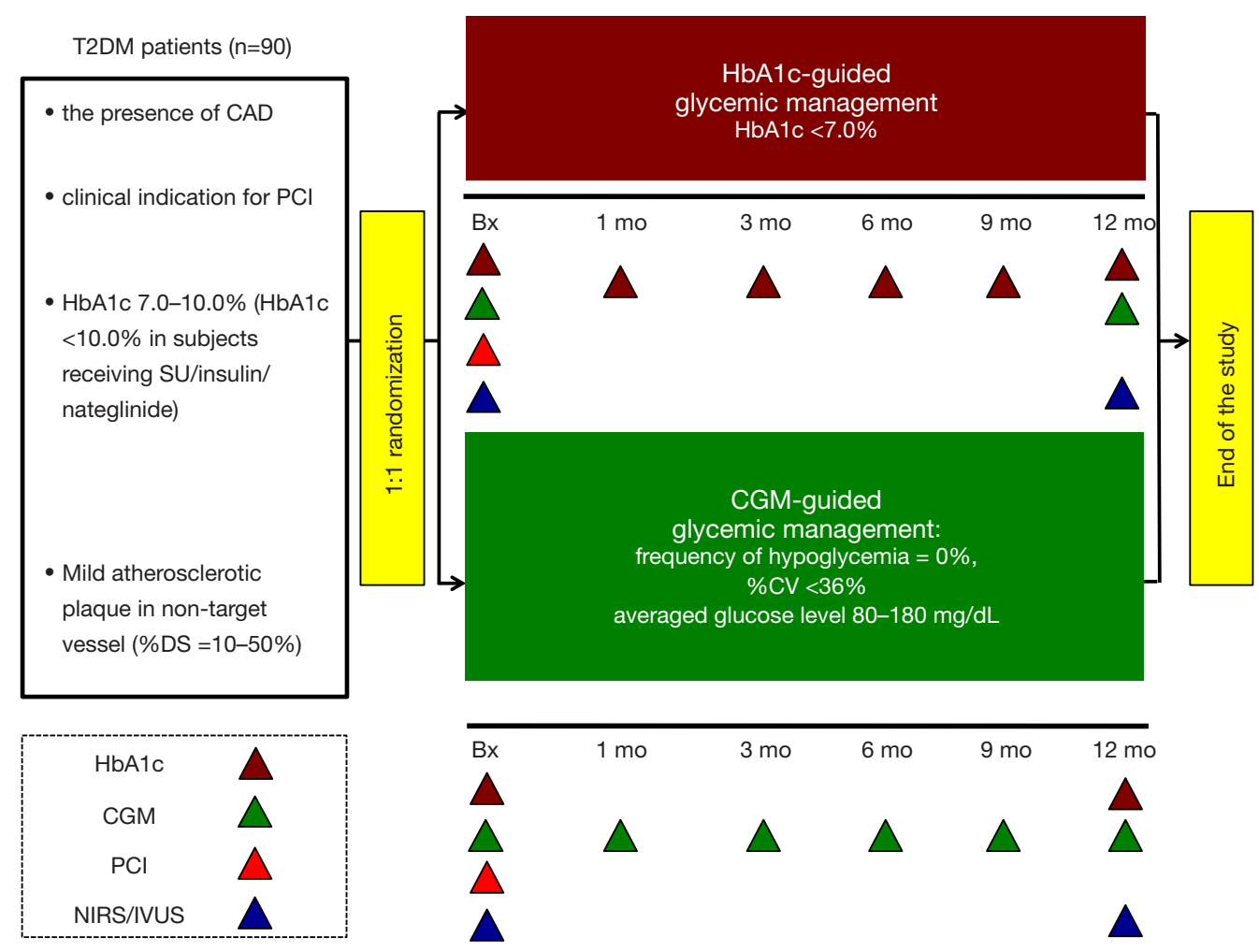

Figure 1 Study design of the OPTIMAL study. Bx, baseline; CAD, coronary artery disease; CGM, continuous glucose monitoring; CV, coefficient of variance; HbAlc, glycated hemoglobin; IVUS, intravascular ultrasound; mo, month; NIRS, near-infrared spectroscopy; PCI, percutaneous coronary intervention; SU, sulphonylurea; T2DM, type 2 diabetes mellitus.

\section{Safety}

Based on the intention-to-treat the entire population, the OPTIMAL study will monitor safety by collecting the following adverse effects (AEs) during the trial: severe AEs regardless as to whether or not there is causal relationship between the AEs and the study; and relevant AEs such as hypoglycemia, bleeding and pains related to CGM use. The grade of severity, procedures, outcomes, and relationship to the study drug will be evaluated. The principal investigator will promptly report AEs to the study secretariat and to the Data and Safety Monitoring Board (DSMB), which consist of authorized endocrinologists, cardiologists, or neurologist with relevant expertise. The incident of withdrawal from the study will be also reported to the DSMB. The DSMB will then assess the incident and report the decision to the primary investigator. Auditing will be conducted annually.

\section{Analysis of primary and secondary endpoints}

Efficacy and safety analyses will be performed on the full analysis set, which includes all randomized patients. The primary analysis will be performed when all randomized subjects either have completed the scheduled study visits or have withdrawn early from the study. Analysis of the primary endpoint will be conducted with a linear mixed effects model. Analysis will be based on the intention-to-treat principle and patients will contribute to the group to which they were randomized. Patients with missing data for the primary end-point will be excluded from the primary analysis. The secondary analysis will be performed in the same manner as the primary analysis. All comparisons are planned and all $\mathrm{P}$ values will be two sided. $\mathrm{P}$ values $<0.05$ will be considered statistically significant. All statistical analyses will be performed using SAS software version 9.4 (SAS Institute, Cary, NC, USA).

\section{Safety analyses}

For analyses of safety outcomes, missing data will not be imputed, and adverse events will be summarized by treatment group using descriptive statistics. For analyses of 
clinical endpoints, incidence of events will be summarized for each randomized treatment group.

\section{Results}

The primary endpoint is the normalized absolute change in TAV from baseline to 12 months. The secondary endpoints include (I) the absolute change in percent atheroma volume, (II) the percent change in lipid core burden index, (III) the change in coefficient variance measured by CGM, (IV) the association of change in atherogenic markers (proprotein convertase subxilisin/kexin type 9 , fatty-acid binding protein 4 and 5, and caspase-3 and myeloperoxidase) with the normalized absolute change in TAV, $(\mathrm{V})$ change in TAV under the use of specific anti-diabetic agents (dipeptidyl peptidase-4 inhibitors, sodium-glucose transport protein 2 inhibitors and glucagon-like peptide-1 agonists), and (VI) the frequency of hypoglycemia. NIRS/IVUS analysis is performed by two independent physicians who are blinded to patients' information. The matched arterial segment, defined by the presence of a proximal and distal side branch, will be evaluated. With regard to IVUS analysis, in each cross-sectional image, the leading edges of the lumen and EEM will be manually traced. Lipid core burden index on NIRS imaging will be measured by commercially available software (QIvus ${ }^{\circledR}$; Medis, Leiden, the Netherlands).

This is a superiority trial powered for the primary endpoint. Our previous randomized clinical trial (UMIN0000107) reported $24.7 \%$ reduction of coronary atheroma progression rate in early-stage type 2 diabetic patients with optimal control of glycemic variability, reflected by achieving a lower level of postprandial glucose (7). Considering that the response to antiatherosclerotic therapies is diminished in the advanced type 2 diabetic subjects $(8,9)$, we assume this anti-atherosclerotic efficacy would be half in our T2DM subjects. We estimate that $12.4 \%$ reduction of progression rate would occur in advanced T2DM patients who achieved better glycemic variability. $12.4 \%$ angiographical reduction of progression rate would correspond to $37.05 \mathrm{~mm}^{3}$ more reduction in atheroma volume (10). By adopting a common SD of 59, a total sample size of 82 patients would provide statistical power of $80 \%$ at a significance level of $5 \%$ for a two-sided $t$ test. Anticipating a dropout rate of $10 \%$ at 12 months after PCI, a total of 90 patients should be recruited (45 per arm).

\section{Discussion}

Accumulating evidence suggests the importance of glycemic variability in the occurrence of macrovascular disease in T2DM subjects. Abrupt glycemic surge has been shown to induce oxidative stress and enhance endothelial cell apoptosis more potently compared to sustained hyperglycemia in T2DM patients $(11,12)$. These atherogenic properties of glycemic variability have been supported by epidemiological and observational studies which consistently reported the association of diabetic macrovascular diseases with postprandial but not fasting hyperglycemia (13-16). In addition to these glycemic surges, hypoglycemia has been also shown to promote progression of atherosclerosis via triggering inflammation and endothelial dysfunctions $(17,18)$. Collectively, these findings indicate the need for the clinically-applicable tool to accurately visualize glucose fluctuation and then select adequate glucose-lowering agents in subjects with T2DM.

The usefulness of CGM for the glycemic management of T2DM has been already reported by recent randomized controlled trials. CGM-based glycemic management has enabled to quantitatively evaluate glycemic variability, improve glycemic control and reduce the risk of hypoglycemia (6). Additionally, the mean amplitude of glycemic excursion, one of CGM-derived measures for glycemic variability, has been shown to associate with the progression of coronary atherosclerosis and its instability $(19,20)$. This finding indicates the potential of CGM-based glycemic management to effectively modify the extent of glucose fluctuation, which may potentially lead to slowing atheroma progression.

The current study will employ a novel intravascular imaging catheter which combine intravascular ultrasound (IVUS) and near-infrared spectroscopy (NIRS). Serial IVUS imaging has been pivotal approach in outlining the anti-atherosclerotic effects of medical therapies within the arterial wall (21). NIRS imaging enables to quantitatively measure the degree of lipidic materials within coronary atheroma in vivo, which associates with future coronary events (22). The combination of IVUS and NIRS will provide additive information whether CGM-based glycemic management has any influence on both the quantity and quality of coronary atheroma in T2DM patients with CAD.

\section{Conclusions}

In conclusion, T2DM patients with $\mathrm{CAD}$ are still the highrisk subjects who more frequently have macrovascular diseases despite guideline-recommended medical therapies. The collaboration of CGM use with serial NIRS/IVUS imaging will explore whether less atheroma progression 
can be achieved under CGM-based glycemic management, compared to HbAlc-based approach. These data will serve as an important prelude to the future clinical outcome studies testing the efficacy of anti-glycemic therapies with CGM.

\section{Acknowledgments}

The authors thank Miss Maki Maruki and Miss Emi Kanai for their kind and excellent assistance at the Department of Cardiovascular Medicine and the Division of Atherosclerosis and Diabetes of the National Cerebral and Cardiovascular Center.

Funding: This trial is supported by Japan Agency for Medical Research and Development (grant number: 18ek0210104h0001).

\section{Footnote}

Conflicts of Interest: The authors have no conflicts of interest to declare.

Ethical Statement: The protocol was approved by the institutional ethics committees (M30-152), and written informed consent will be obtained from all study participants prior to entering the study. The authors are accountable for all aspects of the work in ensuring that questions related to the accuracy or integrity of any part of the work are appropriately investigated and resolved.

\section{References}

1. Davies MJ, D'Alessio DA, Fradkin J, et al. Management of hyperglycemia in type 2 diabetes, 2018. A consensus report by the American Diabetes Association (ADA) and the European Association for the Study of Diabetes (EASD). Diabetologia 2018;61:2461.

2. American Diabetes Association. 6. Glycemic Targets: Standards of Medical Care in Diabetes-2019. Diabetes Care 2019;42:S61-S70.

3. Action to Control Cardiovascular Risk in Diabetes Study Group, Gerstein HC, Miller ME, et al. Effects of intensive glucose lowering in type 2 diabetes. $\mathrm{N}$ Engl J Med 2008;358:2545-59.

4. ADVANCE Collaborative Group, Patel A, MacMahon $\mathrm{S}$, et al. Intensive blood glucose control and vascular outcomes in patients with type 2 diabetes. $\mathrm{N}$ Engl J Med 2008;358:2560-72.

5. Duckworth W, Abraira C, Moritz T, et al. Glucose control and vascular complications in veterans with type 2 diabetes. N Engl J Med 2009;360:129-39.

6. Danne T, Nimri R, Battelino T, et al. International Consensus on Use of Continuous Glucose Monitoring. Diabetes Care 2017;40:1631-40.

7. Kataoka Y, Yasuda S, Miyamoto Y, et al. Effects of voglibose and nateglinide on glycemic status and coronary atherosclerosis in early-stage diabetic patients. Circ J 2012;76:712-20.

8. Nicholls SJ, Tuzcu EM, Kalidindi S, et al. Effect of diabetes on progression of coronary atherosclerosis and arterial remodeling: a pooled analysis of 5 intravascular ultrasound trials. J Am Coll Cardiol 2008;52:255-62.

9. Kataoka Y, Hammadah M, Puri R, et al. Plaque microstructures in patients with coronary artery disease who achieved very low low-density lipoprotein cholesterol levels. Atherosclerosis 2015;242:490-5.

10. Ballantyne CM, Raichlen JS, Nicholls SJ, et al. Effect of rosuvastatin therapy on coronary artery stenoses assessed by quantitative coronary angiography: a study to evaluate the effect of rosuvastatin on intravascular ultrasound-derived coronary atheroma burden. Circulation 2008;117:2458-66.

11. Monnier L, Mas E, Ginet C, et al. Activation of oxidative stress by acute glucose fluctuations compared with sustained chronic hyperglycemia in patients with type 2 diabetes. JAMA 2006;295:1681-7.

12. Buscemi S, Re A, Batsis JA, et al. Glycaemic variability using continuous glucose monitoring and endothelial function in the metabolic syndrome and in type 2 diabetes. Diabet Med 2010;27:872-8.

13. Esposito K, Nappo F, Marfella R, et al. Inflammatory cytokine concentrations are acutely increased by hyperglycemia in humans: Role of oxidative stress. Circulation 2002;106: 2067-72.

14. Cavalot F, Pagliarino A, Valle M, et al. Postprandial blood glucose predicts cardiovascular events and all-cause mortality in type 2 diabetes in a 14-year follow-up: lessons from the San Luigi Gonzaga Diabetes Study. Diabetes Care 2011;34:2237-43.

15. Meigs JB, Nathan DM, D'Agostino RB Sr, et al. Fasting and postchallenge glycemia and cardiovascular disease risk: the Framingham Offspring Study. Diabetes Care 2002;25:1845-50.

16. DECODE Study Group. Glucose tolerance and mortality: comparison of WHO and American Diabetes Association diagnostic criteria. The DECODE study group. European Diabetes Epidemiology Group. Diabetes Epidemiology: 
Collaborative analysis Of Diagnostic criteria in Europe. Lancet 1999;354:617-21.

17. Yakubovich N, Gerstein HC. Serious cardiovascular outcomes in diabetes: The role of hypoglycemia. Circulation 2011;123:342-8.

18. Saremi A, Bahn GD, Reaven PD. A Link Between Hypoglycemia and Progression of Atherosclerosis in the Veterans Affairs Diabetes Trial (VADT). Diabetes Care 2016;39:448-54.

19. Gohbara M, Hibi K, Mitsuhashi T, et al. Glycemic Variability on Continuous Glucose Monitoring System Correlates With Non-Culprit Vessel Coronary Plaque Vulnerability in Patients With First-Episode Acute Coronary Syndrome - Optical Coherence Tomography
Study. Circ J 2016;80:202-10.

20. Kataoka S, Gohbara M, Iwahashi N, et al. Glycemic Variability on Continuous Glucose Monitoring System Predicts Rapid Progression of Non-Culprit Lesions in Patients With Acute Coronary Syndrome. Circ J 2015;79:2246-54.

21. Kataoka Y, Andrews J, Puri R, et al. Plaque burden, microstructures and compositions underachieving very low LDL-C levels. Curr Opin Endocrinol Diabetes Obes 2017;24:122-32.

22. Kataoka Y, Puri R, Andrews J, et al. In vivo visualization of lipid coronary atheroma with intravascular near-infrared spectroscopy. Expert Rev Cardiovasc Ther 2017;15:775-85.

Cite this article as: Kataoka Y, Hosoda K, Makino H, Matsubara M, Matsuo M, Ohata Y, Koezuka R, Tamanaha T, Tomita T, Honda-Kohmo K, Noguchi M, Son C, Nishimura K, Asaumi Y, Miyamoto Y, Noguchi T, Yasuda S. The efficacy of glycemic control with continuous glucose monitoring on atheroma progression: rationale and design of the Observation of Coronary Atheroma Progression under Continuous Glucose Monitoring Guidance in Patients with Type 2 Diabetes Mellitus (OPTIMAL). Cardiovasc Diagn Ther 2019;9(5):431-438. doi: 10.21037/cdt.2019.09.02 
Supplementary

Table S1 Study organization

\begin{tabular}{ll}
\hline Role & Names \\
\hline Principal investigator & Yu Kataoka \\
Steering Committee & $\begin{array}{l}\text { Kiminori Hosoda, Yoshihiro Miyamoto, } \\
\text { Hisashi Makino, Teruo Noguchi, Satoshi } \\
\text { Yasuda }\end{array}$ \\
Executive Committee & $\begin{array}{l}\text { Masaki Matsubara, Miki Matsuo, Yoko } \\
\text { Ohata, Ryo Koezuka, Tamiko Tamanaha, } \\
\text { Tsutomu Tomita, Kyoko Koumo, Michio }\end{array}$ \\
Noguchi, Cheol Son, Yasuhide Asaumi \\
Statistical analysis & $\begin{array}{l}\text { Kunihiro Nishimura } \\
\text { Monitoring }\end{array}$ \\
$\begin{array}{l}\text { Kata center and } \\
\text { management }\end{array}$ & $\begin{array}{l}\text { Satoshi Honda } \\
\text { Data and safety } \\
\text { monitoring board }\end{array}$ \\
$\begin{array}{l}\text { Trials secretariat } \\
\text { Hiroki Yoshihara, Kazuo Washio, Makoto }\end{array}$ \\
\hline
\end{tabular}

